\title{
Characterizations for pseudo core inverses in a ring with involution
}

\author{
Huihui Zhu ${ }^{\mathrm{a}, \mathrm{b}, *}$, Pedro Patrício ${ }^{\mathrm{b}, \mathrm{c}}$ \\ ${ }^{a}$ School of Mathematics, Hefei University of Technology, Hefei 230009, China. \\ ${ }^{b}$ CMAT-Centro de Matemática, Universidade do Minho, Braga 4710-057, Portugal. \\ ${ }^{c}$ Departamento de Matemática e Aplicações, Universidade do Minho, Braga 4710-057, \\ Portugal.
}

\begin{abstract}
Let $R$ be a unital $*$-ring. In this paper, we give several characterizations for the pseudo core and dual pseudo core inverses of an element by units in $R$. Moreover, their relations with $*$-DMP are obtained. As a special case, the existence criteria for EP elements ( $*$-DMP with index 1$)$ are given. Then, the double commutant property of hybrid $(b, c)$-inverses is considered. Finally, the relations of the inverse along an element and the hybrid $(b, c)$-inverse, the (dual) pseudo core inverses and the hybrid $(b, c)$-inverse are investigated.

Keywords: pseudo core inverses, Drazin inverses, Moore-Penrose inverses, *-DMP, hybrid $(b, c)$-inverses, double commutant property 2010 MSC: 15A09, 16U99
\end{abstract}

\section{Introduction}

Since Moore-Penrose inverses and Drazin inverses were introduced, there comes up lots of generalized inverses. For instance, *-DMP [14], DMP inverses [10], Core-EP inverses [11], $m$-EP matrices [9] the inverse along an element [12], hybrid $(b, c)$-inverses [3], (dual) core inverses [1, 17] and so on. Many papers $[2,6,13,19,21]$ devoted to the study of these types of generalized inverses. Recently, Gao and Chen [7] introduced the notions of (dual) pseudo core inverses in a ring, as extensions of (dual) core inverses.

\footnotetext{
${ }^{*}$ Corresponding author

Email addresses: hhzhu@hfut.edu.cn (Huihui Zhu), pedro@math.uminho.pt (Pedro Patrício)
} 
In this paper, we aim to investigate $*$-DMP, (dual) pseudo core inverses and hybrid $(b, c)$-inverses. The paper is organized as follows. In Section 2, we give the definitions, notations and basic properties of several generalized inverses. In Section 3, we derive an existence criterion of the pseudo core and dual pseudo core inverses of an element, extending the corresponding results of core and dual core inverses. Pseudo core inverses are further studied using a direct sum of ideals and annihilators. Also, both the pseudo core and dual pseudo core inverses of an element with its $*$-DMP inverses are considered. In Section 4, $\{1,3\}$-inverses, $\{1,4\}$-inverses and pseudo core inverses are further studied by the direct sum of ideals and annihilators. In Section 5, we prove that the hybrid $(b, c)$-inverse shares the generalized double commutant property, and $a$ is invertible along $d$ if and only if it is hybrid $(d, d)$-invertible. As an immediate application, the generalized double commutant property of the inverse along an element is given, extending the double commutant property of Mary in [12]. Furthermore, it is shown in a ring that $a$ is pseudo core invertible if and only if there exists some positive integer $n$ such that $a$ is hybrid $\left(a^{n},\left(a^{n}\right)^{*}\right)$-invertible. Finally, the generalized double commutant property of the pseudo core inverse is given.

\section{Preliminaries}

In this section, we mainly introduce some notations, and give some fundamental results of generalized inverses, which will be useful in the sequent sections.

Let $R$ be a unital ring. An element $a \in R$ is (von Neumann) regular if there exists an $x \in R$ such that $a=a x a$. Such an $x$ is called an inner inverse or $\{1\}$-inverse of $a$, and is denoted by $a^{-}$.

Definition 2.1. [5] Let $R$ be a unital ring. An element $a \in R$ is said to have $a$ Drazin inverse if there exists an element $b \in R$ and a nonnegative integer $k$ such that

$$
\text { (i) } a^{k}=a^{k+1} b \text {, (ii) } b a b=b \text {, (iii) } a b=b a \text {. }
$$

The element $b$ satisfying the above conditions (i)-(iii) is unique if it exists, and is denoted by $a^{D}$. The smallest nonnegative integer $k$ is called the Drazin index of $a$ and is denoted by $\operatorname{ind}(a)$. 
We call $a \in R$ group invertible if it is Drazin invertible and $\operatorname{ind}(a)=1$. Also, the group inverse of $a$ is unique and is denoted by $a^{\#}$. Moreover, $a \in R$ is group invertible if and only if $a \in a^{2} R \cap R a^{2}$. In this case, $a^{\#}=a x^{2}=$ yax $=y^{2} a$, where $x, y \in R$ satisfy $a=a^{2} x=y a^{2}$. By $R^{D}, R^{\#}$ we denote the sets of all Drazin invertible, group invertible elements in $R$, respectively.

In what follows, we assume that $R$ is a unital $*$-ring, i.e. a ring with unity 1 and an involution $*$ satisfying $\left(x^{*}\right)^{*}=x,(x y)^{*}=y^{*} x^{*}$ and $(x+y)^{*}=x^{*}+y^{*}$ for all $x, y \in R$.

Definition 2.2. [15] Let $a \in R$. An element $a \in R$ is called Moore-Penrose invertible if there exists an element $x \in R$ such that the following four equations hold

$$
\text { (i) } a x a=a, \quad \text { (ii) } x a x=x, \text { (iii) }(a x)^{*}=a x, \quad \text { (iv) }(x a)^{*}=x a \text {. }
$$

The element $x$ satisfying the equations (i)-(iv) is called a Moore-Penrose inverse of $a$. Such an $x$ is unique if it exists, and is denoted by $a^{\dagger}$.

We call $a$ to be $\{1,3\}$-invertible if $x$ satisfies the equations (i) and (iii) in Definition 2.2. Such an $x$ is called a $\{1,3\}$-inverse of $a$, and is denoted by $a^{(1,3)}$. Similarly, $a$ is $\{1,4\}$-invertible if $x$ satisfies the equations (i) and (iv). Such an $x$ is called a $\{1,4\}$-inverse of $a$, and is denoted by $a^{(1,4)}$. By $R^{\dagger}, R^{(1,3)}$ and $R^{(1,4)}$ we denote the sets of all Moore-Penrose invertible, $\{1,3\}$-invertible and $\{1,4\}$-invertible elements in $R$, respectively.

It is known [22] that $a \in R^{(1,3)}$ if and only if $a \in R a^{*} a$. If $a=y a^{*} a$ for some $y \in R$, then $y^{*}$ is a $\{1,3\}$-inverse of $a$. Dually, $a \in R^{(1,4)}$ if and only if $a \in a a^{*} R$. If $a=a a^{*} x$ for some $x \in R$, then $x^{*}$ is a $\{1,4\}$-inverse of $a$. It is well known that $a \in R^{\dagger}$ if and only if $a \in a a^{*} R \cap R a^{*} a$.

Definition 2.3. [7] Let $a \in R$. We call a pseudo core invertible if there exist $x \in R$ and a positive integer $n$ such that

$$
\text { (i) } x a^{n+1}=a^{n} \text {, (ii) } a x^{2}=x \text {, (iii) }(a x)^{*}=a x \text {. }
$$

The element $x$ satisfying the above conditions is called a pseudo core inverse of $a$. It is unique if it exists, and is denoted by $a^{\mathbb{D}}$. The smallest positive integer $n$ is called the pseudo core index of $a$, and is denoted by $\mathrm{I}(a)$. 
The dual pseudo core inverse of $a$, when exists, is defined as the unique $a_{\mathbb{D}}$ such that $a^{m+1} a_{\mathbb{Q}}=a^{m},\left(a_{\mathbb{O}}\right)^{2} a=a_{\mathbb{Q}}$ and $\left(a_{\mathbb{D}} a\right)^{*}=a_{\mathbb{Q}} a$ for some positive integer $m$. In this case, the smallest positive integer $m$ satisfying the conditions above is called the dual pseudo core index of $a$, and is denoted by $\mathrm{I}^{\prime}(a)$. We denote by $R^{\mathbb{D}}, R_{\mathbb{S}}$ the sets of all pseudo core invertible, dual pseudo core invertible elements in $R$, respectively.

\section{Characterizations for (dual) pseudo core inverses by units}

We begin with some lemmas, which play an important role in the sequel. As usual, the symbol $R^{-1}$ denotes the set of all invertible elements of $R$.

Lemma 3.1. Let $a, b \in R$. Then

(i) If $1+a b \in R^{-1}$, then so is $1+b a$ and $(1+b a)^{-1}=1-b(1+a b)^{-1} a$.

(ii) If $a b-1 \in R^{-1}$, then so is $b a-1$ and $(b a-1)^{-1}=b(a b-1)^{-1} a-1$.

The two formulae in Lemma 3.1 above are well known as Jacobson's Lemma.

Lemma 3.2. Let $a \in R$ be regular and let $k$ be a positive integer. Then the following conditions are equivalent:

(i) $a \in R^{\#}$.

(ii) $a$ is regular and $u=a^{k}-1+a a^{-} \in R^{-1}$.

(iii) $a$ is regular and $v=a^{k}-1+a^{-} a \in R^{-1}$.

(iv) $a$ is regular and $s=a^{k}+1-a a^{-} \in R^{-1}$.

(v) $a$ is regular and $t=a^{k}+1-a^{-} a \in R^{-1}$.

In this case, $a^{\#}=x^{-1} a^{2 k-1} y^{-1}$, where $x \in\{u, s\}$ and $y \in\{v, t\}$.

Proof. It follows from Lemma 3.1 that (ii) $\Leftrightarrow$ (iii) and (iv) $\Leftrightarrow$ (v). Also, (i) $\Leftrightarrow$ (iv) was proved in [2]. Similarly, we can get (i) $\Leftrightarrow$ (ii).

We next give the existence criterion of the Drazin inverse of an element in rings, which is essentially the same as [16, p. 107].

Recall that $a \in R^{D}$ with $\operatorname{ind}(a)=n$ if and only if $n$ is the smallest positive integer such that $a^{n} \in R^{\#}$. Moreover, $a^{D}=a^{n-1}\left(a^{n}\right)^{\#}$. Taking $k=1$ in Lemma 3.2, one can get that $a^{n} \in R^{\#}$ if and only if $a^{n}$ is regular and $a^{n} \pm e \in R^{-1}$ if and only if $a^{n}$ is regular and $a^{n} \pm f \in R^{-1}$, where $e=1-a^{n}\left(a^{n}\right)^{-}$and $f=1-\left(a^{n}\right)^{-} a^{n}$. Hence, we have 
Lemma 3.3. Let $a \in R$. Then the following conditions are equivalent:

(i) $a \in R^{D}$ with ind $(a)=n$.

(ii) $n$ is the smallest positive integer such that $a^{n}$ is regular and $u=$ $a^{n}+1-a^{n}\left(a^{n}\right)^{-} \in R^{-1}$.

(iii) $n$ is the smallest positive integer such that $a^{n}$ is regular and $v=$ $a^{n}+1-\left(a^{n}\right)^{-} a^{n} \in R^{-1}$.

(iv) $n$ is the smallest positive integer such that $a^{n}$ is regular and $u=$ $a^{n}-1+a^{n}\left(a^{n}\right)^{-} \in R^{-1}$.

(v) $n$ is the smallest positive integer such that $a^{n}$ is regular and $v=$ $a^{n}-1+\left(a^{n}\right)^{-} a^{n} \in R^{-1}$.

Lemma 3.4. [20, Theorem 2.3] Let $a \in R$ be regular. Then

(i) $a \in R^{\dagger}$.

(ii) $u=a a^{*}+1-a a^{-}$is left or right invertible.

(iii) $u^{\prime}=a a^{*}-1+a a^{-}$is left or right invertible.

(iv) $v=a^{*} a+1-a^{-} a$ is left or right invertible.

(v) $v=a^{*} a-1+a^{-} a$ is left or right invertible.

In this case, $a^{\dagger}=\left(u_{l}^{-1} a\right)^{*}=\left(\left(u_{l}^{\prime}\right)^{-1} a\right)^{*}=\left(v_{r}^{-1} a\right)^{*}=\left(\left(v_{r}^{\prime}\right)^{-1} a\right)^{*}$, where $u_{l}^{-1}, v_{r}^{-1}$ denote a left inverse of $u$, a right inverse of $v$, respectively.

Lemma 3.5. [21, Theorem 3.12] Let $a \in R$. Then $a \in R^{\dagger}$ if and only if $a \in a a^{*} a R$ if and only if $a \in R a a^{*} a$. In particular, if $a=a a^{*} a x$ or $a=y a a^{*} a$ for some $x, y \in R$, then $a^{\dagger}=(a x)^{*}=(y a)^{*}$.

Lemma 3.6. [7, Theorem 2.3] Let $a \in R$. Then $a \in R^{\circledR}$ with $I(a)=m$ if and only if $a \in R^{D}$ with $\operatorname{ind}(a)=m$ and $a^{n} \in R^{(1,3)}$ whenever $n \geq m$. Moreover, $a^{\mathbb{D}}=a^{D} a^{n}\left(a^{n}\right)^{(1,3)}$.

Similarly, we can show that $a \in R_{\mathbb{D}}$ with $\mathrm{I}^{\prime}(a)=m$ if and only if $a \in$ $R^{D}$ with $\operatorname{ind}(a)=m$ and $a^{n} \in R^{(1,4)}$ whenever $n \geq m$. Moreover, $a_{\mathbb{B}}=$ $\left(a^{n}\right)^{(1,4)} a^{n} a^{D}$.

One can get from [7] that if $a \in R^{\mathbb{D}} \cap R_{\mathbb{Q}}$ then $\mathrm{I}(a)=\mathrm{I}^{\prime}(a)=\operatorname{ind}(a)$. It is well known that $a \in R^{\dagger}$ if and only if $a \in R^{(1,3)} \cap R^{(1,4)}$. Hence, we claim that $a \in R^{\mathbb{D}} \cap R_{\mathbb{D}}$ with $\mathrm{I}(a)=\mathrm{I}^{\prime}(a)=n$ if and only if $a \in R^{D}$ with $\operatorname{ind}(a)=n$ and $a^{n} \in R^{\dagger}$.

We hence get the following characterizations for both pseudo core invertible and dual pseudo core invertible elements in a $*$-ring. 
Theorem 3.7. Let $a \in R$. Then the following conditions are equivalent:

(i) $a \in R^{\mathbb{D}} \cap R_{\mathbb{O}}$ with $I(a)=I^{\prime}(a)=n$.

(ii) $n$ is the smallest positive integer such that $a^{n}$ is regular and $u=$ $a^{n}\left(a^{n}\right)^{*} a^{n}+1-a^{n}\left(a^{n}\right)^{-} \in R^{-1}$.

(iii) $n$ is the smallest positive integer such that $a^{n}$ is regular and $u^{\prime}=$ $\left(a^{n}\right)^{*} a^{2 n}+1-\left(a^{n}\right)^{-} a^{n} \in R^{-1}$.

(iv) $n$ is the smallest positive integer such that $a^{n}$ is regular and $v=$ $a^{2 n}\left(a^{n}\right)^{*}+1-a^{n}\left(a^{n}\right)^{-} \in R^{-1}$.

(v) $n$ is the smallest positive integer such that $a^{n}$ is regular and $v^{\prime}=$ $a^{n}\left(a^{n}\right)^{*} a^{n}+1-\left(a^{n}\right)^{-} a^{n} \in R^{-1}$.

(vi) $n$ is the smallest positive integer such that $a^{n}$ is regular and $s=$ $a^{n}\left(a^{n}\right)^{*} a^{n}-1+a^{n}\left(a^{n}\right)^{-} \in R^{-1}$.

(vii) $n$ is the smallest positive integer such that $a^{n}$ is regular and $s^{\prime}=$ $\left(a^{n}\right)^{*} a^{2 n}-1+\left(a^{n}\right)^{-} a^{n} \in R^{-1}$.

(viii) $n$ is the smallest positive integer such that $a^{n}$ is regular and $t=$ $a^{n}\left(a^{n}\right)^{*} a^{n}-1+\left(a^{n}\right)^{-} a^{n} \in R^{-1}$.

(ix) $n$ is the smallest positive integer such that $a^{n}$ is regular and $t^{\prime}=$ $a^{2 n}\left(a^{n}\right)^{*}-1+a^{n}\left(a^{n}\right)^{-} \in R^{-1}$.

In this case, $a^{\mathbb{Q}}=a^{n-1} u^{-1} a^{n}\left(a^{n}\right)^{*}$ and $a_{\mathbb{\Phi}}=\left(v^{-1} a^{2 n}\right)^{*} a^{2 n-1}\left(a^{n}\right)^{*} a^{n} t^{-1}$.

Proof. It follows from Lemma 3.1 above that (ii) $\Leftrightarrow$ (iii), (iv) $\Leftrightarrow$ (v), (vi) $\Leftrightarrow$ (vii), and (viii) $\Leftrightarrow$ (ix). Next, it is sufficient to prove (i) $\Leftrightarrow$ (ii) $\Leftrightarrow$ (iv) $\Leftrightarrow$ (vi) $\Leftrightarrow$ (viii).

(i) $\Rightarrow$ (ii) As $a \in R^{\mathbb{D}} \cap R_{\mathbb{D}}$ with $\mathrm{I}(a)=\mathrm{I}^{\prime}(a)=n \Leftrightarrow a \in R^{D}$ with $\operatorname{ind}(a)=n$ and $a^{n} \in R^{\dagger}$, then, by Lemma 3.3, $n$ is the smallest positive integer such that $a^{n}$ is regular and $a^{n}+1-a^{n}\left(a^{n}\right)^{-} \in R^{-1}$. Since $a^{n} \in R^{\dagger}$, we have $a^{n}\left(a^{n}\right)^{*}+1-a^{n}\left(a^{n}\right)^{-} \in R^{-1}$ by Lemma 3.4. Again, applying Lemma 3.1, it follows $a^{n}\left(a^{n}\right)^{*} a^{n}\left(a^{n}\right)^{-}+1-a^{n}\left(a^{n}\right)^{-} \in R^{-1}$. So, $\left(a^{n}\left(a^{n}\right)^{*} a^{n}\left(a^{n}\right)^{-}+\right.$ $\left.1-a^{n}\left(a^{n}\right)^{-}\right)\left(a^{n}+1-a^{n}\left(a^{n}\right)^{-}\right)=a^{n}\left(a^{n}\right)^{*} a^{n}+1-a^{n}\left(a^{n}\right)^{-}=u \in R^{-1}$.

(ii) $\Rightarrow$ (iv) Since $u=a^{n}\left(a^{n}\right)^{*} a^{n}+1-a^{n}\left(a^{n}\right)^{-} \in R^{-1}$, we have $u^{\prime}=$ $\left(a^{n}\right)^{*} a^{2 n}+1-\left(a^{n}\right)^{-} a^{n} \in R^{-1}$ and $a^{n} u^{\prime}=a^{n}\left(a^{n}\right)^{*} a^{2 n}$, and consequently $a^{n}=a^{n}\left(a^{n}\right)^{*} a^{2 n}\left(u^{\prime}\right)^{-1} \in a^{n}\left(a^{n}\right)^{*} a^{n} R$. By Lemma 3.5, it gives $a^{n} \in R^{\dagger}$ and $\left(a^{n}\right)^{\dagger}=\left(a^{2 n}\left(u^{\prime}\right)^{-1}\right)^{*}$, which in turn implies $a^{n}\left(a^{n}\right)^{*}+1-a^{n}\left(a^{n}\right)^{-} \in R^{-1}$ by Lemma 3.4. Hence, by Lemma 3.1, $a^{n}\left(a^{n}\right)^{*} a^{n}\left(a^{n}\right)^{-}+1-a^{n}\left(a^{n}\right)^{-} \in R^{-1}$. As $a^{n}+1-a^{n}\left(a^{n}\right)^{-}=\left(a^{n}\left(a^{n}\right)^{*} a^{n}\left(a^{n}\right)^{-}+1-a^{n}\left(a^{n}\right)^{-}\right)^{-1} u \in R^{-1}$, then $a^{2 n}\left(a^{n}\right)^{-}+1-a^{n}\left(a^{n}\right)^{-} \in R^{-1}$. So, $\left(a^{2 n}\left(a^{n}\right)^{-}+1-a^{n}\left(a^{n}\right)^{-}\right)\left(a^{n}\left(a^{n}\right)^{*}+1-\right.$ $\left.a^{n}\left(a^{n}\right)^{-}\right)=v \in R^{-1}$.

(iv) $\Rightarrow$ (vi) and (vi) $\Rightarrow$ (ix) By a similar proof of (ii) $\Rightarrow$ (iv). 
(ix) $\Rightarrow$ (i) We have $a^{n}=\left(t^{\prime}\right)^{-1} a^{2 n}\left(a^{n}\right)^{*} a^{n}$, and by Lemma 3.5, $a^{n} \in R^{\dagger}$. So, $a^{n}\left(a^{n}\right)^{*}-1+a^{n}\left(a^{n}\right)^{-} \in R^{-1}$. As $a^{2 n}\left(a^{n}\right)^{-}+1-a^{n}\left(a^{n}\right)^{-}=t^{\prime}\left(a^{n}\left(a^{n}\right)^{*}-\right.$ $\left.1+a^{n}\left(a^{n}\right)^{-}\right)^{-1} \in R^{-1}$, then, by Lemma 3.1, $a^{n}+1-a^{n}\left(a^{n}\right)^{-} \in R^{-1}$. It follows from Lemma 3.3 that $a \in R^{D}$ with ind $(a)=n$, which together with $a^{n} \in R^{\dagger}$ imply $a \in R^{\mathbb{D}} \cap R_{\mathbb{D}}$ with $\mathrm{I}(a)=\mathrm{I}^{\prime}(a)=\operatorname{ind}(a)=n$.

Note the fact that $a \in R^{D}$ with $\operatorname{ind}(a)=n$ if and only if $n$ is the smallest positive integer such that $a^{n} \in R^{\#}$. As $u a^{n}=a^{n}\left(a^{n}\right)^{*} a^{2 n}$, then $a^{n}=$ $u^{-1} a^{n}\left(a^{n}\right)^{*} a^{2 n}$, which together with $a^{n} \in R^{\#}$ give $\left(a^{n}\right)^{\#}=\left(u^{-1} a^{n}\left(a^{n}\right)^{*}\right)^{2} a^{n}$. Thus, $a^{D}=a^{n-1}\left(u^{-1} a^{n}\left(a^{n}\right)^{*}\right)^{2} a^{n}$.

Therefore, we have

$$
\begin{aligned}
a^{\mathbb{D}} & =a^{D} a^{n}\left(a^{n}\right)^{(1,3)}=a^{n-1}\left(u^{-1} a^{n}\left(a^{n}\right)^{*}\right)^{2} a^{n} a^{n}\left(a^{n}\right)^{(1,3)} \\
& =a^{n-1} u^{-1} a^{n}\left(a^{n}\right)^{*} u^{-1} a^{n}\left(a^{n}\right)^{*} a^{2 n}\left(a^{n}\right)^{(1,3)}=a^{n-1} u^{-1} a^{n}\left(a^{n}\right)^{*} a^{n}\left(a^{n}\right)^{(1,3)} \\
& =a^{n-1} u^{-1} a^{n}\left(a^{n}\right)^{*}\left(a^{n}\left(a^{n}\right)^{(1,3)}\right)^{*}=a^{n-1} u^{-1} a^{n}\left(a^{n}\left(a^{n}\right)^{(1,3)} a^{n}\right)^{*} \\
& =a^{n-1} u^{-1} a^{n}\left(a^{n}\right)^{*} .
\end{aligned}
$$

Dually, by $a^{D}=a^{2 n-1}\left(\left(a^{n}\right)^{*} a^{n} t^{-1}\right)^{2}$ and $\left(a^{n}\right)^{(1,4)}=\left(v^{-1} a^{2 n}\right)^{*}$, we obtain $a_{\text {过 }}=\left(a^{n}\right)^{(1,4)} a^{n} a^{D}=\left(a^{n}\right)^{(1,4)} a^{n} a^{n-1}\left(a^{n}\right)^{*} a^{n} t^{-1}=\left(v^{-1} a^{2 n}\right)^{*} a^{2 n-1}\left(a^{n}\right)^{*} a^{n} t^{-1}$.

Set $n=1$ in Definition 2.3, the pseudo core inverse with $\mathrm{I}(a)=1$ is just the core inverse. Analogously, the dual core inverse is the dual pseudo core inverse with $\mathrm{I}^{\prime}(a)=1$. They are denoted by the unique $a^{\oplus}$ and $a_{\oplus}$, respectively. We denote by $R^{\oplus}$ and $R_{\circledast}$ the sets of all core invertible and dual core invertible elements in $R$, respectively.

Set $\mathrm{I}(a)=\mathrm{I}^{\prime}(a)=n=1$ in Theorem 3.7. Then $a_{\mathbb{D}}=\left(a^{n}\right)^{(1,4)} a^{n} a^{n-1}\left(a^{n}\right)^{*} a^{n} t^{-1}$ can be reduced to $a_{\circledast}=a^{*} a t^{-1}$ Indeed, $a^{(1,4)} a a^{*} a t^{-1}=\left(a^{(1,4)} a\right)^{*} a^{*} a t^{-1}=$ $\left(a a^{(1,4)} a\right)^{*} a t^{-1}=a^{*} a t^{-1}$. Hence, we have

Corollary 3.8. [2, Theorem 5.6] Let $a \in R$ be regular. Then the following conditions are equivalent:

(i) $a \in R^{\oplus} \cap R_{\oplus}$.

(ii) $u=a a^{*} a+1-a a^{-} \in R^{-1}$.

(iii) $u^{\prime}=a^{*} a^{2}+1-a^{-} a \in R^{-1}$.

(iv) $v=a^{2} a^{*}+1-a a^{-} \in R^{-1}$.

(v) $v^{\prime}=a a^{*} a+1-a^{-} a \in R^{-1}$.

(vi) $s=a a^{*} a-1+a a^{-} \in R^{-1}$.

(vii) $s^{\prime}=a^{*} a^{2}-1+a^{-} a \in R^{-1}$. 
(viii) $t=a a^{*} a-1+a^{-} a \in R^{-1}$.

(ix) $t^{\prime}=a^{2} a^{*}-1+a a^{-} \in R^{-1}$.

In this case, $a^{\boxplus}=u^{-1} a a^{*}$ and $a_{\circledast}=a^{*} a t^{-1}$.

An element $a \in R$ is called $*$-DMP with index $n$ (see [14, Definition 6]) if $n$ is the smallest positive integer such that $\left(a^{n}\right)^{\#}$ and $\left(a^{n}\right)^{\dagger}$ both exist and $\left(a^{n}\right)^{\#}=\left(a^{n}\right)^{\dagger}$. In particular, the $*$-DMP with index 1 is well known as EP elements, i.e. $*$-gMP invertible elements (see [14, Definition 1]).

Note that $a \in R^{\boxplus} \cap R_{\circledast}$ if and only if $a \in R^{\#} \cap R^{\dagger}$. Then we have

Proposition 3.9. Let $a \in R$. Then $a \in R^{\mathbb{D}} \cap R_{\mathbb{O}}$ with $I(a)=I^{\prime}(a)=n$ if and only if $n$ is the smallest positive integer such that $a^{n} \in R^{\boxplus} \cap R_{\circledast}$.

We remark that $*$-DMP of index $n$ is in the intersection of pseudo core inverse and dual pseudo core inverse of $a$, where $\mathrm{I}(a)=\mathrm{I}^{\prime}(a)=n$. It would be of interest to give conditions for $a \in R^{\mathbb{D}} \cap R_{\mathbb{D}}$ to be $*$-DMP? The following theorem gives their equivalence. Herein, a lemma is given.

Lemma 3.10. [14, Proposition 2] Let $a \in R$. Then

(i) If $a R=a^{*} R$, then $a \in R^{\#}$ if and only if $a \in R^{\dagger}$. In this case, $a^{\#}=a^{\dagger}$.

(ii) If $a^{\#}=a^{\dagger}$, then $a R=a^{*} R$.

Theorem 3.11. Let $a \in R$. Then the following conditions are equivalent:

(i) $a \in R^{\mathbb{D}} \cap R_{\mathbb{D}}$ with $I(a)=I^{\prime}(a)=n$ and $a a^{D}=\left(a a^{D}\right)^{*}$.

(ii) $a$ is $*$-DMP with index $n$.

Proof. (i) $\Rightarrow$ (ii) As $a \in R^{\mathbb{D}} \cap R_{\mathbb{D}}$ with $\mathrm{I}(a)=n$, then $a \in R^{D}$ with $\operatorname{ind}(a)=n$ and $n$ is the smallest positive integer such that $a^{n} \in R^{\#}$.

It is well known that $a a^{D}=\left(a a^{D}\right)^{n}=\left(a^{D}\right)^{n} a^{n}$. Since $a^{n}=a a^{D} a^{n}=$ $\left(a a^{D}\right)^{*} a^{n}=\left(\left(a a^{D}\right)^{n}\right)^{*} a^{n}=\left(a^{n}\right)^{*}\left(\left(a^{D}\right)^{n}\right)^{*} a^{n}$, we have $a^{n} R \subseteq\left(a^{n}\right)^{*} R$. Also, note that $a^{n}=a^{n}\left(\left(a^{D} a\right)^{n}\right)^{*}=a^{n}\left(\left(a^{D}\right)^{n}\right)^{*}\left(a^{n}\right)^{*}$. Then, applying the involution $*$ on the equality above, we get $\left(a^{n}\right)^{*} R \subseteq a^{n} R$. So, $a^{n} R=\left(a^{n}\right)^{*} R$.

As $\left(a^{n}\right)^{\#}$ exists, then by Lemma 3.10(i), $a^{n} \in R^{\dagger}$ and $\left(a^{n}\right)^{\dagger}=\left(a^{n}\right)^{\#}$, i.e. $a$ is $*$-DMP with index $n$.

(ii) $\Rightarrow$ (i) As $a$ is $*$-DMP with index $n$, then $n$ is the smallest positive integer such that $\left(a^{n}\right)^{\#}=\left(a^{n}\right)^{\dagger}$, and by Proposition 3.9, $a \in R^{\mathbb{D}} \cap R_{\mathbb{O}}$ with $I(a)=I^{\prime}(a)=n$. Note that $a^{D}=a^{n-1}\left(a^{n}\right)^{\#}=a^{n-1}\left(a^{n}\right)^{\dagger}$. Then, $a a^{D}=a^{n}\left(a^{n}\right)^{\dagger}=\left(a a^{D}\right)^{*}$. 
Corollary 3.12. Let $a \in R$. Then the following conditions are equivalent:

(i) $a \in R^{\oplus} \cap R_{\oplus}$ and $a a^{\#}=\left(a a^{\#}\right)^{*}$.

(ii) a is EP.

Applying Lemmas 3.4 and 3.10, we present characterizations of EP elements by one-sided invertibility.

Corollary 3.13. Let $a \in R$ be regular. Then the following conditions are equivalent:

(i) $a$ is $E P$.

(ii) $a R=a^{*} R$ and $u=a a^{*}+1-a a^{-}$is left or right invertible.

(iii) $a R=a^{*} R$ and $u^{\prime}=a a^{*}-1+a a^{-}$is left or right invertible.

(iv) $a R=a^{*} R$ and $v=a^{*} a+1-a^{-} a$ is left or right invertible.

(v) $a R=a^{*} R$ and $v=a^{*} a-1+a^{-} a$ is left or right invertible.

\section{Characterizations for (dual) pseudo core inverses by direct sums}

In this section, we aim to derive the characterization for (dual) pseudo core inverses of $a \in R$.

By the symbols ${ }^{0} a=\{x \in R: x a=0\}$ and $a^{0}=\{y \in R: a y=0\}$ we denote the sets of all left annihilators and right annihilators of $a$ in $R$, respectively. Hartwig [8] derived that $a \in R^{\#}$ if and only if $R=a R \oplus a^{0}$ if and only if $R=R a \oplus^{0} a$. Inspired by Hartwig's results, we give existence criteria for the Drazin inverse of $a$ in $R$.

Proposition 4.1. Let $a \in R$. Then the following conditions are equivalent:

(i) $a \in R^{D}$.

(ii) $R=a^{n} R \oplus\left(a^{n}\right)^{0}$, for some positive integer $n$.

(iii) $R=R a^{n} \oplus^{0}\left(a^{n}\right)$, for some positive integer $n$.

In this case, $a^{D}=a^{2 n-1} b^{2}$, where $b \in R$ and $c \in\left(a^{n}\right)^{0}$ satisfy $1=a^{n} b+c$.

Proof. (i) $\Rightarrow$ (ii) As $a \in R^{D}$, then there exists a positive integer $n$ such that $a^{n}=a^{n+1} a^{D}$, which gives $a^{n}\left(1-a a^{D}\right)=0$, i.e. $1-a a^{D} \in\left(a^{n}\right)^{0}$. Then for any $r \in R$, we have $r=\left(a a^{D}+1-a a^{D}\right) r=a a^{D} r+\left(1-a a^{D}\right) r=$ $a^{n}\left(a^{D}\right)^{n} r+\left(1-a a^{D}\right) r \in a^{n} R+\left(a^{n}\right)^{0}$. We next show $a^{n} R \cap\left(a^{n}\right)^{0}=0$. Given any $b \in a^{n} R \cap\left(a^{n}\right)^{0}$, then $b=a^{n} x$ for some $x \in R$ and $a^{n} b=0$. Hence, $b=a^{n} x=a^{n+1} a^{D} x=a a^{D} a^{n} x=\left(a a^{D}\right)^{n} b=\left(a^{D}\right)^{n} a^{n} b=0$. So, $R=a^{n} R \oplus\left(a^{n}\right)^{0}$ for some positive integer $n$. 
(ii) $\Rightarrow$ (i) Note that $R=a^{n} R \oplus\left(a^{n}\right)^{0}$ for some positive integer $n$. Then there exists some $b \in R$ and $c \in\left(a^{n}\right)^{0}$ such that $1=a^{n} b+c$, and consequently $a^{2 n}=a^{2 n} b a^{n}$, i.e. $a^{n}\left(a^{n}-a^{n} b a^{n}\right)=0$. So, $a^{n}=a^{n} b a^{n}$ since $a^{n}-a^{n} b a^{n} \in$ $a^{n} R \cap\left(a^{n}\right)^{0}=0$. Moreover, we claim $\left(a^{n}\right)^{0} \subseteq\left(a^{n} b\right)^{0}$. Indeed, for any $s=\left(a^{n}\right)^{0}$, it follows $a^{2 n} b s=a^{n} s=0$ and hence $a^{n} b s \in a^{n} R \cap\left(a^{n}\right)^{0}=0$. So, $s \in\left(a^{n} b\right)^{0}$. As $a^{n}-b a^{2 n} \in\left(a^{n}\right)^{0} \subseteq\left(a^{n} b\right)^{0}$, then $a^{n} b^{2} a^{2 n}=a^{n} b a^{n}=a^{n}$, which together with $a^{n}=a^{2 n} b$ imply $a^{n} \in R^{\#}$ and $\left(a^{n}\right)^{\#}=a^{n} b^{2}$. Therefore, $a \in R^{D}$ with $a^{D}=a^{2 n-1} b^{2}$.

(i) $\Leftrightarrow$ (iii) It is similar to the proof of (i) $\Leftrightarrow$ (ii).

We next give characterizations for $\{1,3\}$-inverses and $\{1,4\}$-inverses of $a^{k}$ in $R$, where $k$ is a positive integer.

Proposition 4.2. Let $a \in R$ and let $n$ be a positive integer. Then $a^{n} \in R^{(1,3)}$ if and only if $R=R\left(a^{n}\right)^{*} \oplus^{0}\left(a^{n}\right)$.

Proposition 4.3. Let $a \in R$ and let $n$ be a positive integer. Then $a^{n} \in R^{(1,4)}$ if and only if $R=\left(a^{n}\right)^{*} R \oplus\left(a^{n}\right)^{0}$.

Applying Propositions 4.1 and 4.2, we obtain characterizations for the pseudo core inverse of an element.

Theorem 4.4. Let $a \in R$. Then the following conditions are equivalent:

(i) $a \in R^{\Phi}$.

(ii) $R=R a^{n} \oplus^{0}\left(a^{n}\right)=R\left(a^{n}\right)^{*} \oplus^{0}\left(a^{n}\right)$, for some positive integer $n$.

(iii) $R=a^{n} R \oplus\left(a^{n}\right)^{0}=R\left(a^{n}\right)^{*} \oplus^{0}\left(a^{n}\right)$, for some positive integer $n$.

In this case, $a^{\mathbb{D}}=a^{2 n-1} b^{2} a^{n} s^{*}$, where $b, s \in R, c \in\left(a^{n}\right)^{0}$ and $t \in{ }^{0}\left(a^{n}\right)$ satisfy $1=a^{n} b+c=s\left(a^{n}\right)^{*}+t$.

Dually, we can give the characterization of the dual pseudo core inverse.

Theorem 4.5. Let $a \in R$. Then the following conditions are equivalent:

(i) $a \in R_{\mathbb{D}}$.

(ii) $R=R a^{n} \oplus^{0}\left(a^{n}\right)=\left(a^{n}\right)^{*} R \oplus\left(a^{n}\right)^{0}$, for some positive integer $n$.

(iii) $R=a^{n} R \oplus\left(a^{n}\right)^{0}=\left(a^{n}\right)^{*} R \oplus\left(a^{n}\right)^{0}$, for some positive integer $n$.

In this case, $a_{\mathbb{D}}=q^{*} a^{3 n-1} g^{2}$, where $g, q \in R$ and $h, r \in\left(a^{n}\right)^{0}$ satisfy $1=a^{n} g+h=\left(a^{n}\right)^{*} q+r$.

We combine Theorems 4.4 and 4.5 to obtain the following existence criterion of both pseudo core invertible and dual pseudo core invertible elements. 
Corollary 4.6. Let $a \in R$. Then the following conditions are equivalent:

(i) $a \in R^{\Phi} \cap R_{\mathbb{D}}$.

(ii) $R=R a^{n} \oplus^{0}\left(a^{n}\right)=\left(a^{n}\right)^{*} R \oplus\left(a^{n}\right)^{0}=R\left(a^{n}\right)^{*} \oplus^{0}\left(a^{n}\right)$, for some positive integer $n$.

(iii) $R=a^{n} R \oplus\left(a^{n}\right)^{0}=\left(a^{n}\right)^{*} R \oplus\left(a^{n}\right)^{0}=R\left(a^{n}\right)^{*} \oplus^{0}\left(a^{n}\right)$, for some positive integer $n$.

We next give brief characterizations for the pseudo core and dual pseudo core inverses by the direct sum of ideals and annihilators in a ring.

Theorem 4.7. Let $a \in R$. Then the following conditions are equivalent:

(i) $a \in R^{\mathbb{D}} \cap R_{\mathbb{D}}$.

(ii) $R=a^{n} R \oplus\left(a^{n}\right)^{0}=\left(a^{n}\right)^{*} a^{n} R \oplus\left(a^{n}\right)^{0}$, for some positive integer $n$.

(iii) $R=a^{n} R \oplus\left(a^{n}\right)^{0}=R a^{n}\left(a^{n}\right)^{*} \oplus^{0} a^{n}$, for some positive integer $n$.

(iv) $R=R a^{n} \oplus^{0}\left(a^{n}\right)=R a^{n}\left(a^{n}\right)^{*} \oplus^{0} a^{n}$, for some positive integer $n$.

(v) $R=R a^{n} \oplus^{0}\left(a^{n}\right)=R a^{n}\left(a^{n}\right)^{*} \oplus^{0} a^{n}$, for some positive integer $n$.

Proof. It is sufficient to prove (i) $\Leftrightarrow$ (ii) $\Leftrightarrow$ (iii). The rest follows by noting that $a \in R^{D} \Leftrightarrow R=R a^{n} \oplus^{0}\left(a^{n}\right)$, for some positive integer $n$.

(i) $\Rightarrow$ (ii) Suppose $a \in R^{\mathbb{D}} \cap R_{\mathbb{D}}$ with $\mathrm{I}(a)=\mathrm{I}^{\prime}(a)=n$. Then $a \in R^{D}$ with $\operatorname{ind}(a)=n$ and $a^{n} \in R^{\dagger}$. As $a \in R^{D}$ with ind $(a)=n$, then, by Proposition 4.1, $R=a^{n} R \oplus\left(a^{n}\right)^{0}$.

It follows from Lemma 3.5 that $a^{n} \in R^{\dagger}$ implies $a^{n}=a^{n}\left(a^{n}\right)^{*} a^{n} x$ for some $x \in R$. Set $s:=1-\left(a^{n}\right)^{*} a^{n} x$. Then $1=\left(a^{n}\right)^{*} a^{n} x+s \in\left(a^{n}\right)^{*} a^{n} R+\left(a^{n}\right)^{0}$. We next show $\left(a^{n}\right)^{*} a^{n} R \cap\left(a^{n}\right)^{0}=0$. Let $t \in\left(a^{n}\right)^{*} a^{n} R \cap\left(a^{n}\right)^{0}$. Then there is $y \in R$ such that $t=\left(a^{n}\right)^{*} a^{n} y=\left(a^{n}\left(a^{n}\right)^{\dagger} a^{n}\right)^{*} a^{n} y=\left(a^{n}\right)^{\dagger} a^{n}\left(a^{n}\right)^{*} a^{n} y=\left(a^{n}\right)^{\dagger} a^{n} t=0$ since $a^{n} t=0$. Thus, $R=\left(a^{n}\right)^{*} a^{n} R \oplus\left(a^{n}\right)^{0}$.

So, $R=a^{n} R \oplus\left(a^{n}\right)^{0}=\left(a^{n}\right)^{*} a^{n} R \oplus\left(a^{n}\right)^{0}$, for some positive integer $n$.

(ii) $\Rightarrow$ (i) It is obvious that $a \in R^{D}$ by Proposition 4.1. Since $R=$ $\left(a^{n}\right)^{*} a^{n} R \oplus\left(a^{n}\right)^{0}$, we have $a^{n} R=a^{n}\left(a^{n}\right)^{*} a^{n} R$ and hence $a^{n} \in R^{\dagger}$ by Lemma 3.5. As $a \in R^{D}$ and $a^{n} \in R^{\dagger} \Leftrightarrow a^{n} \in R^{(1,3)} \cap R^{(1,4)}$, then, by Lemma 3.6, $a \in R^{\mathbb{D}} \cap R_{\mathbb{D}}$.

(i) $\Leftrightarrow$ (iii) It is analogous to the proof of (i) $\Leftrightarrow$ (ii) by noting $a^{n} \in R^{\dagger} \Leftrightarrow$ $a^{n} \in R a^{n}\left(a^{n}\right)^{*} a^{n}$. 


\section{Relations with other types of generalized inverses and its prop- erty}

Let $a, d \in R$. Following [12], an element $a$ is called invertible along $d$ if there exists some $y \in R$ such that $y a y=y, y R=d R$ and $R y=R d$. Such a $y$ is called the inverse of $a$ along $d$. It is unique, and is denoted by $a^{\| d}$.

In 2012, Drazin [3] introduced a class of outer generalized inverses called hybrid $(b, c)$-inverses. Let $a, b, c \in R$ and let $r^{0}=\{x \in R: r x=0\}$ denote all right annihilators of $r \in R$. An element $a$ is called hybrid $(b, c)$-invertible if there exists an element $y \in R$ such that yay $=y, y R=b R$ and $y^{0}=c^{0}$. Such a $y$ is unique if it exists, and is called the hybrid $(b, c)$-inverse of $a$. More results on hybrid $(b, c)$-inverses can be referred to [18].

Lemma 5.1. (see e.g. [17]) Let $b, y \in R$. Then

(i) If $y R=b R$, then ${ }^{0} y={ }^{0} b$.

(ii) If $R y=R b$, then $y^{0}=b^{0}$.

For any $a, d \in R$, by Lemma 5.1 above, if $a$ is invertible along $d$, then it is hybrid $(d, d)$-invertible. We next illustrate that the converse also holds.

Proposition 5.2. Let $a, d \in R$. Then a is invertible along $d$ if and only if a is hybrid $(d, d)$-invertible. Moreover, the inverse of a along $d$ coincides with the hybrid $(d, d)$-inverse of a.

Proof. We only need prove the "if" part.

Let $y \in R$ be the hybrid $(d, d)$-inverse of $a$. Then yay $=y$, and consequently $1-a y \in y^{0} \subseteq d^{0}$. So, $R d=R d a y \subseteq R y$. Since $y R=d R$, we have $y=d r$ for some $r \in R$. Hence, $y=y a y=d r a y$. Also, $y R=d R$ gives $d=y s$ for some $s \in R$. So, Multiplying $y=d r a y$ by $s$ on the right yields $d=d r a d$. Hence, $d$ is regular, which combines with $d^{0} \subseteq y^{0}$ to conclude $R y \subseteq R d$. So, $R y=R d$ and $y$ is the inverse of $a$ along $d$.

Drazin [4] showed that the $(b, c)$-inverse shares the generalized double commutant property. We next shows that the hybrid $(b, c)$-inverse also satisfies this property.

Theorem 5.3. Let $a_{i}, b_{i}, c_{i}, y_{i} \in R$ and let $y_{i}$ be the hybrid $\left(b_{i}, c_{i}\right)$-inverse of $a_{i}(i=1,2)$. Then for any $x \in R, x a_{1}=a_{2} x, x b_{1}=b_{2} x$ and $x c_{1}=c_{2} x$ imply $x y_{1}=y_{2} x$. 
Proof. Since $y_{2}$ is the hybrid $\left(b_{2}, c_{2}\right)$-inverse of $a_{2}$, it follows $y_{2} R=b_{2} R$. Then there exists some $z \in R$ such that $b_{2}=y_{2} z$, which together with $y_{2} a_{2} y_{2}=y_{2}$ gives $y_{2} a_{2} b_{2}=b_{2}$. Thus, we have $x b_{1}=b_{2} x=y_{2} a_{2} b_{2} x=y_{2} a_{2} x b_{1}$, which implies $x-y_{2} a_{2} x \in{ }^{0} b_{1}$. Note that $y_{1} R=b_{1} R$. Then, by Lemma 5.1, ${ }^{0} y_{1}={ }^{0} b_{1}$. So, $x-y_{2} a_{2} x \in{ }^{0} y_{1}$, and hence $x y_{1}=y_{2} a_{2} x y_{1}$.

Note that $y_{1} a_{1} y_{1}=y_{1}$ concludes $1-a_{1} y_{1} \in y_{1}^{0}=c_{1}^{0}$. Then $c_{1}=c_{1} a_{1} y_{1}$ and $c_{2} x=x c_{1}=x c_{1} a_{1} y_{1}=c_{2} x a_{1} y_{1}=c_{2} a_{2} x y_{1}$. Consequently, $x-a_{2} x y_{1} \in$ $c_{2}^{0}=y_{2}^{0}$, i.e. $y_{2} x=y_{2} a_{2} x y_{1}$.

Therefore, $x y_{1}=y_{2} x$.

Applying Proposition 5.2 and Theorem 5.3, we have the following corollaries.

Corollary 5.4. Let $a_{1}, a_{2}, d, x \in R$ with $x a_{1}=a_{2} x$ and $x d=d x$. If $a_{1}^{\| d}$ and $a_{2}^{\| d}$ exist, then $x a_{1}^{\| d}=a_{2}^{\| d} x$.

Corollary 5.5. Let $a, d_{1}, d_{2}, x \in R$ with $x a=a x$ and $x d_{1}=d_{2} x$. If $a^{\| d_{1}}$ and $a^{\| d_{2}}$ exist, then $x a^{\| d_{1}}=a^{\| d_{2}} x$.

In particular, if we take $a_{1}=a_{2}$ or $d_{1}=d_{2}$ in the above corollaries, then we get the classical double commutant property of the inverse along an element.

Corollary 5.6. [12, Theorem 10] Let $a, d, x \in R$ with $x a=a x$ and $x d=d x$. If $a^{\| d}$ exists, then $x a^{\| d}=a^{\| d} x$.

We next consider the relations between the pseudo core inverse and the hybrid $(b, c)$-inverse in a ring.

Theorem 5.7. Let $a, x \in R$. Then $x$ is the pseudo core inverse of $a$ if and only if it is the hybrid $\left(a^{n},\left(a^{n}\right)^{*}\right)$-inverse of a, for some positive integer $n$.

Proof. Suppose that $x$ is the pseudo core inverse of $a$ with $\mathrm{I}(a)=n$. Then, by the definition, we have $x a^{n+1}=a^{n}, a x^{2}=x$ and $(a x)^{*}=a x$.

We next show that $x$ is the hybrid $\left(a^{n},\left(a^{n}\right)^{*}\right)$-inverse of $a$ by proving $x a x=x, x R=a^{n} R$ and $x^{0}=\left(\left(a^{n}\right)^{*}\right)^{0}$.

(a) Note that $x=a x^{2}=a\left(a x^{2}\right) x=a^{2} x^{3}=\cdots=a^{n} x^{n+1}$. Then $x a x=$ $x a\left(a x^{2}\right)=x a^{2} x^{2}=\cdots=x a^{n+1} x^{n+1}=\left(x a^{n+1}\right) x^{n+1}=a^{n} x^{n+1}=x$.

(b) $x R=a^{n} R$ since $a^{n} R=x a^{n+1} R \subseteq x R$, and $x R=a^{n} x^{n+1} R \subseteq a^{n} R$. 
(c) Let $s \in x^{0}$, i.e. $x s=0$. Then $\left(a^{n}\right)^{*} s=\left(x a^{n+1}\right)^{*} s=\left(a^{n+1}\right)^{*} x^{*} s=$ $\left(a^{n+1}\right)^{*}\left(a x^{2}\right)^{*} s=\left(a^{n+1}\right)^{*} x^{*}(a x)^{*} s=\left(a^{n+1}\right)^{*} x^{*} a x s=0$. So, $x^{0} \subseteq\left(\left(a^{n}\right)^{*}\right)^{0}$.

For the converse, as $x R=a^{n} R$, then $R x^{*}=R\left(a^{n}\right)^{*}$, which implies $x^{*}=$ $p\left(a^{n}\right)^{*}$ for some $p \in R$. Thus, for any $t \in\left(\left(a^{n}\right)^{*}\right)^{0}$, we get, by the equality (a), $x t=x a x t=x(a x)^{*} t=x x^{*} a^{*} t=x p\left(a^{n}\right)^{*} a^{*} t=x p a^{*}\left(a^{n}\right)^{*} t=0$. That is $x^{0}=\left(\left(a^{n}\right)^{*}\right)^{0}$.

Conversely, let $x$ be the hybrid $\left(a^{n},\left(a^{n}\right)^{*}\right)$-inverse of $a$. Then $x a x=x$, $x R=a^{n} R$ and $x^{0}=\left(\left(a^{n}\right)^{*}\right)^{0}$.

As $x R=a^{n} R$, then $a^{n}=x b$ for some $b \in R$. Multiplying $x a x=x$ by $b$ on the right yields $x a^{n+1}=a^{n}$.

Since $1-a x \in x^{0}=\left(\left(a^{n}\right)^{*}\right)^{0}$, we have $\left(a^{n}\right)^{*}=\left(a^{n}\right)^{*} a x$ and hence $a^{n}=$ $(a x)^{*} a^{n}$. Also, $x a x=x$ and $x R=a^{n} R$ imply $x \in a^{n} R x$. Then there exists $c \in R$ such that $x=a^{n} c x$, and consequently $a x=a^{n+1} c x=a^{n} a c x=$ $(a x)^{*} a^{n} a c x=(a x)^{*} a\left(a^{n} c x\right)=(a x)^{*} a x$. So, $(a x)^{*}=a x$.

Note that $a^{n}=(a x)^{*} a^{n}$ and $(a x)^{*}=a x$. Then $a^{n}=a x a^{n}$. We have $a x^{2}=a x\left(a^{n} c x\right)=\left(a x a^{n}\right) c x=a^{n} c x=x$.

Therefore, $x$ is the pseudo core inverse of $a$.

Remark 5.8. By the proof of Theorem 5.7, we claim that if $a \in R^{\mathbb{D}}$ with $I(a)=n$, then $a$ is also hybrid $\left(a^{m},\left(a^{m}\right)^{*}\right)$-invertible for any positive integer $m \geq n$.

Corollary 5.9. Let $a, x \in R$. Then $x$ is the core inverse of $a$ if and only if it is the hybrid $\left(a, a^{*}\right)$-inverse of $a$.

Dually, we have

Theorem 5.10. Let $a, y \in R$. Then $y$ is the dual pseudo core inverse of a if and only if it is the hybrid $\left(\left(a^{n}\right)^{*}, a^{n}\right)$-inverse of a, for some positive integer $n$.

On returning to our last argument of the generalized double commutant property of pseudo core inverses.

Proposition 5.11. Let $a_{1}, a_{2}, x \in R$ with $x a_{1}=a_{2} x$ and $x a_{1}^{*}=a_{2}^{*} x$. If $a_{1}, a_{2} \in R^{\mathbb{D}}$, then $x a_{1}^{\mathbb{D}}=a_{2}^{\mathbb{D}} x$. 
Proof. Let $a_{1} \in R^{\mathbb{D}}$ with $\mathrm{I}\left(a_{1}\right)=m$ and let $a_{2} \in R^{\mathbb{D}}$ with $\mathrm{I}\left(a_{2}\right)=n$. By Theorem 5.7, we know that $a_{1}$ is $\left(a_{1}^{m},\left(a_{1}^{*}\right)^{m}\right)$-invertible, and $a_{2}$ is $\left(a_{2}^{n},\left(a_{2}^{*}\right)^{n}\right)$ invertible.

Let $k=\max \{m, n\}$. Then, from Remark 5.8, $a_{i}$ is $\left(a_{i}^{k},\left(a_{i}^{*}\right)^{k}\right)$-invertible $(i=1,2)$. By induction, we get $x a_{1}^{k}=a_{2}^{k} x$ and $x\left(a_{1}^{k}\right)^{*}=\left(a_{2}^{k}\right)^{*} x$. It follows from Theorem 5.3 that $x a_{1}^{\mathbb{D}}=a_{2}^{\mathbb{D}} x$.

Corollary 5.12. [7, Proposition 4.2] Let $a, x \in R$ with $x a=$ ax and $x a^{*}=$ $a^{*} x$. If $a \in R^{\mathbb{D}}$, then $x a^{\mathbb{D}}=a^{\mathbb{D}} x$.

Corollary 5.13. [2, Theorem 3.2] Let $a_{1}, a_{2}, x \in R$ with $x a_{1}=a_{2} x$ and $x a_{1}^{*}=a_{2}^{*} x$. If $a_{1}, a_{2} \in R^{\oplus}$, then $x a_{1}^{\oplus}=a_{2}^{\oplus} x$.

\section{ACKNOWLEDGMENTS}

The authors are highly grateful to the referee for his/her valuable comments and suggestions which greatly improved this paper. This research is supported by the Fundamental Research Funds for the Central Universities, the Natural Science Foundation of Anhui Province (No. 1808085QA16) and the Portuguese Funds through FCT- 'Fundação para a Ciência e a Tecnologia', within the project UID-MAT-00013/2013.

\section{References}

[1] O.M. Baksalary, G. Trenkler, Core inverse of matrices, Linear Multilinear Algebra 58 (2010) 681-697.

[2] J.L. Chen, H.H. Zhu, P. Patricio and Y.L. Zhang, Characterizations and representations of core and dual core inverses, Canad. Math. Bull. 60 (2017) 269-282.

[3] M.P. Drazin, A class of outer generalized inverses, Linear Algebra Appl. 436 (2012) 1909-1923.

[4] M.P. Drazin, Commuting properties of generalized inverses, Linear Multilinear Algebra 61 (2013) 1675-1681.

[5] M.P. Drazin, Pseudo-inverses in associative rings and semigroups, Amer. Math. Monthly 65 (1958) 506-514. 
[6] D.E. Ferreyra, F.E. Levis, N. Thome, Revisiting the core EP inverse and its extension to rectangular matrices, Quaest. Math. (2017) DOI: 10.2989/16073606.2017.1377779

[7] Y.F. Gao, J.L. Chen, Pseudo core inverses in rings with involution, Comm. Algebra 46 (2018) 38-50.

[8] R. E. Hartwig, Block generalized inverses. Arch. Rational Mech. Anal. 61 (1976) 197-251.

[9] S.B. Malik, L. Rueda, N. Thome, The class of $m$-EP and $m$-normal matrices, Linear Multilinear Algebra 64 (2016) 2119-2132.

[10] S.B. Malik, N. Thome, On a new generalized inverse for matrices of an arbitrary index, Appl. Math. Comput. 226 (2014) 575-580.

[11] K. Manjunatha Prased, K.S. Mohana, Core-EP inverse, Linear Multilinear Algebra 62 (2014) 792-802.

[12] X. Mary, On generalized inverses and Green's relations, Linear Algebra Appl. 434 (2011) 1836-1844.

[13] X. Mary, P. Patrício, Generalized inverses modulo $\mathcal{H}$ in semigroups and rings, Linear Multilinear Algebra 61 (2013) 1130-1135.

[14] P. Patrício, R. Puystjens, Drazin-Moore-Penrose invertibility in rings, Linear Algebra Appl. 389 (2004) 159-173.

[15] R. Penrose, A generalized inverse for matrices, Proc. Camb. Phil. Soc. 51 (1955) 406-413.

[16] R. Puystjens, M.C. Gouveia, Drazin invertibility for matrices over an arbitrary ring, Linear Algebra Appl. 385 (2004) 105-116.

[17] D.S. Rakić, N.C. Dinčić, D.S. Djordjević, Group, Moore-Penrose, core and dual core inverse in rings with involution, Linear Algebra Appl. 463 (2014) 115-133.

[18] H.H. Zhu, Further results on several types of generalized inverses, Comm. Algebra (2018) DOI: 10.1080/00927872.2017.1412450 
[19] H.H. Zhu, On DMP inveres and $m$-EP elements in rings, Linear Multilinear Algebra (2018) DOI: 10.1080/03081087.2018.1432546

[20] H.H. Zhu, Several characterizations for generalized inverses in a ring, Linear Multilinear Algebra (2018) DOI: 10.1080/03081087.2017.1353948

[21] H.H. Zhu, J.L. Chen, P. Patrício, X. Mary, Centralizer's applications to the inverse along an element, Appl. Math. Comput. 315 (2017) 27-33.

[22] H.H. Zhu, X.X. Zhang, J.L. Chen, Centralizers and their applications to generalized inverses, Linear Algebra Appl. 458 (2014) 291-300. 